\title{
On Significant Crisp Representatives of Fuzzy Regions in Colour Images
}

\author{
B. Prados-Suárez, D. Sánchez, and J. Chamorro-Martínez \\ Department of Computer Science and Artificial Intelligence, University of Granada \\ C/ Periodista Daniel Saucedo Aranda s/n, 18071 Granada, Spain \\ e-mail : \{belenps,daniel,jesus\}@decsai.ugr.es
}

\begin{abstract}
In this paper we propose a technique to obtain a set of crisp representatives of an imprecise region in color images. In our approach, we start by obtaining a fuzzy model of the region by employing a fuzzy region growing procedure. The set of crisp representatives is obtained from that model as the set of $\alpha$-cuts corresponding to maximal variations of homogeneity. Several experiments and a comparison with both crisp and fuzzy techniques are provided, showing the suitability of the proposal against other approaches.
\end{abstract}

Keywords: Image segmentation, fuzzy segmentation, pathbased segmentation, fuzzy connectivity, fuzzy colour homogeneity, crisp representatives.

\section{INTRODUCTION}

Image segmentation, i.e., the process of partitioning the image into regions consisting of sets of connected and homogeneous pixels, is a very important task in image analysis. It is on the basis of many relevant applications, like image database retrieval, medical imaging, and robot vision among others.

Though the usual objective of segmentation is to provide a partition of the whole image, in some applications the attention is pointed to a single region. Examples are medical images where specialists are interested in areas corresponding to a tumor or another disease [6], [11], [10]. In those applications some agent, usually an expert user, indicates what region of the image is of interest (commonly specified by one pixel that, in the user's perception, is representative of the region, called a seed). Let us remark that there are many pixels that are representative of a region and the results are to be similar for any of them.

In many applications it is necessary to find a precise boundary for the region. For example, suppose the medical specialist needs to measure the size of a tumor in an image, i.e., the number of pixels of the region corresponding to the tumor. However, in natural environments most of the regions have imprecise boundaries, as occurs in shadows, bright and color gradients. Hence, it is not possible to determine what pixels are and what are not in the region with total accuracy.

The problem of modeling a region with imprecise boundaries is addressed by fuzzy segmentation techniques, that define a region as a fuzzy subset of pixels [2], [20], [16], [8], [5], [19]. But in our case just a fuzzy region is not a good enough output since, though the region is imprecise, and hence the best and most informative model of the region is a fuzzy one, our problem is to determine in a crisp way which pixels correspond to the region and which do not.

To solve the problem of outlining a region with imprecise boundaries, we propose to obtain a fuzzy model of the region, and then to determine a set of crisp representatives, corresponding to the "best" crisp approximations of the fuzzy region. This approach allows us to obtain crisp solutions and, at the same time, to account for the region's inherent imprecision.

In order to illustrate this problem let us consider the image in figure 1, corresponding to the "Spirograph Nebula" taken by the Hubble in 1999. The white point in the center is the star and each color represent a different expansion level caused by ultraviolet radiation and nuclear foil, offering information like the age of the star. In this case it is quite interesting to have a model that represents the star with the whole color variation in the nebula surrounding it and, in addition, to precisely bound the different expansion levels (indicated by arrows in figure $1)$.

Let us remark that the problem of finding a small set of crisp representatives cannot be solved by classical crisp segmentation techniques since they yield a single crisp approximation. In addition, crisp techniques [14], [7] are not well suited to solve the problem of obtaining the model of a single region, in which a single seed is specified, since at least two seeds are needed, and the model obtained depends not only on the seed indicating the region of interest, but of the number and position of the rest of the seeds. Most of the fuzzy extensions of region growing techniques have the same problem [1], [15], [12].

In order to obtain a fuzzy model of the region, we will employ the proposal introduced in [3]. This approach has two advantages. First, the model of a fuzzy region depends only on the region's seed, and it is independent from the number and position of other seeds (in particular, no additional seed is needed in order to obtain the fuzzy model of the region). Second, the technique is able to adapt to the characteristics (degree of imprecision in the boundaries) of the region being modeled.

The question now is, how to obtain the best crisp representatives? In our view, the best way is to employ a set of $\alpha$-cuts of the fuzzy region. The question is, what $\alpha$-cuts are perceptually relevant, i.e., what $\alpha$-cuts determine possible boundaries for the region? Our idea is that boundaries are characterized by a 


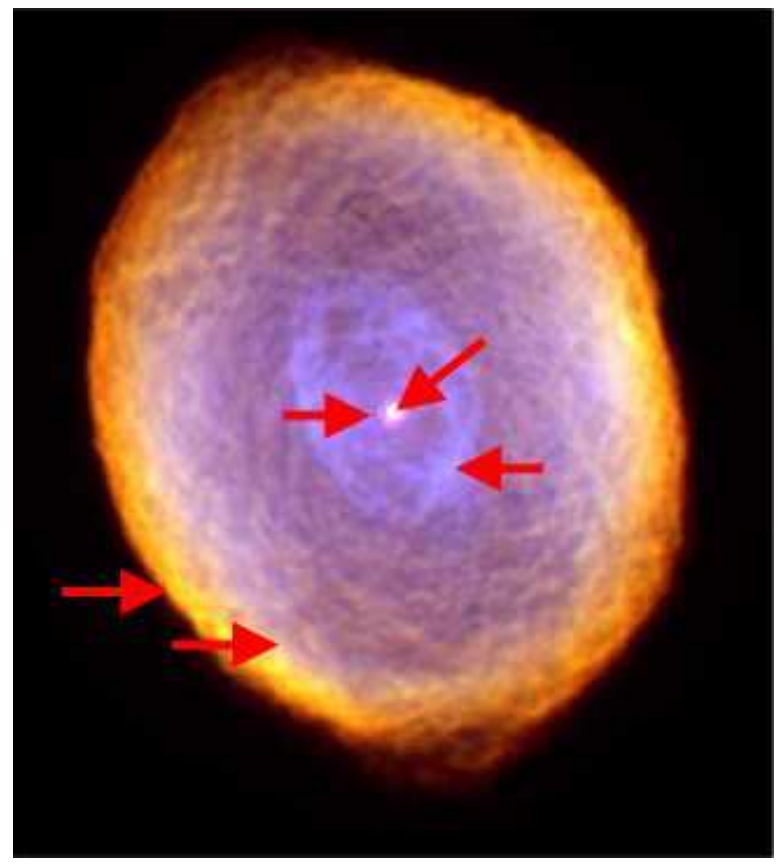

Fig. 1. The Spirograph Nebula image taken by Hubble (NASA and ESA) in 1999. The arrows indicate the intuitive imprecise boundaries of each crisp representative.

change in homogeneity between one side and the other of the boundary. Following this idea, we shall provide a set of crisp representatives as those $\alpha$-cuts where the homogeneity varies significantly.

The paper is organized as follows: in section II we briefly sketch a fuzzy region growing segmentation technique designed for obtaining a fuzzy model suited to the characteristics of the region on the basis of color homogeneity. In section III we propose a measure of homogeneity of crisp representatives of a fuzzy region, and we show a procedure to choose the best crisp approximations. In section IV we show some experiments and we compare our results with those obtained by applying other segmentation techniques, both crisp and fuzzy. Finally, section V contains our conclusions and future work.

\section{Path Based Segmentation}

In this section we briefly summarize the path-based approach presented in [3] to obtain a fuzzy region. This proposal computes the membership degree of a pixel to a region on the basis of the connectivity degree with the seed of the region. To incorporate spatial information about pixel adjacency, connectivity between any pair of pixels is measured as the homogeneity of the most homogeneous path joining them.

\section{A. Pixel Characterization}

Firstly, to characterize a pixel, $p$, in the image, we use a feature vector, $\overrightarrow{f_{p}}=\left[f_{p}^{1}, \ldots, f_{p}^{N}\right]$, where each feature, $f_{p}^{i}$, is a numerical measure of any relevant characteristic obtained for $p$. In our case, we are interested in obtaining homogeneously-colored regions, so we have chosen the human perception based color space HSI (hue, saturation or purity and intensity or lightness) [3]. Hence, the feature vector we use to characterize each pixel $p$ in the image, contains its three band color representation in the HSI color space, as in equation 1:

$$
\overrightarrow{f_{p}}=\left[H_{p}, S_{p}, I_{p}\right]
$$

\section{B. Fuzzy Resemblance Between Pixels}

Once we have characterized each pixel, we use in [3] a resemblance relation, $\mathcal{F} \mathcal{R}$ between feature vectors. The calculation of $\mathcal{F} \mathcal{R}$ depends on the concrete features employed. In our case, it is based on resemblance in colour as given by a normalized distance in the HSI color space, $\triangle C\left(c_{1}, c_{2}\right)$. This distance is defined in [3] on the basis of the differences in $[0,1]$ between color components, $\triangle_{H}, \triangle_{S}$ and $\triangle_{I}$ for hue, saturation and intensity values, respectively.

We define the resemblance between the feature vectors $f_{p}$ and $f_{q}$ corresponding to pixels $p$ and $q$ in the image $\mathcal{I}$ as in equation 2 :

$$
\mathcal{F} \mathcal{R}\left(\overrightarrow{f_{p}}, \overrightarrow{f_{q}}\right)=1-\triangle C\left(\overrightarrow{f_{p}}, \overrightarrow{f_{q}}\right)
$$

Finally, we define a resemblance relation $\mathcal{P} \mathcal{R}$ between neighbor pixels, as the relation $\mathcal{F} \mathcal{R}$ between their corresponding feature vectors, in this way:

$$
\mathcal{P} \mathcal{R}(p, q)=\mathcal{F} \mathcal{R}\left(\overrightarrow{f_{p}}, \overrightarrow{f_{q}}\right)
$$

\section{Fuzzy Connectivity Between Pixels}

So far, we can know the resemblance between two adjacent pixels. To measure resemblance between any two pixels, we use the fuzzy connectivity measure. Fuzzy connectivity of two pixels indicates, in fuzzy path-based image segmentation, the degree to which those pixels belong to a group of topologically connected pixels with resemblant features. Therefore to measure the fuzzy connectivity between two pixels we use information about the homogeneity of the paths joining them.

Given two pixels, $p$ and $q$, a path joining them, $\pi_{p q}$, is a sequence

$$
\pi_{p q}=\left(r_{1}, r_{2}, \ldots, r_{k}\right)
$$

where $k \geq 1$, such that $r_{1}=p$ and $r_{k}=q$ and $r_{i}$ is connected to $r_{i+1} \forall i \in\{1, \ldots, k-1\}$. With $\Pi_{p q}$ we note the set of all the possible paths between $p$ and $q$.

The homogeneity of a path is a function, homo $: \Pi_{p q} \rightarrow$ $[0,1]$, calculated on the basis of resemblance between consecutive points in the path. We deal with homogeneity functions in section II-E. Taking the homo function into account, we define the optimum path between $p$ and $q, \hat{\pi}_{p q}$, as the path linking them with maximum homogeneity. We get the measure of the connectivity between two pixels as the homogeneity value of the optimum path joining them:

$$
\operatorname{conn}(p, q)=\operatorname{homo}\left(\hat{\pi}_{p q}\right)
$$

In practice it is not necessary to evaluate the homogeneity of all the possible paths. An efficient algorithm to obtain the connectivity, linear in both number of pixels and number of seeds, is proposed in [4]. 


\section{Membership Functions for Fuzzy Regions}

The aforementioned fuzzy connectivity between two pixels lets us define, in equation 6 , the membership degree of a pixel $p$, to a region $\widetilde{R_{s}}$, as the connectivity between the pixel and the seed point, $r_{s}$, of the region.

$$
\mu_{\widetilde{R_{s}}}(p)=\operatorname{conn}\left(p, r_{s}\right)
$$

By computing the membership degree of each point $p$ in the image to each region $\widetilde{R_{s}}$, we obtain the set of fuzzy regions resulting of the fuzzy segmentation process, with a computational cost of $O(n)$, with $n$ being the size of the image, as detailed in [4]. In these segmentation process an open problem is the selection of the homogeneity function for each region, depending on its homogeneity characteristics.

\section{E. Homogeneity Functions}

Intuitively a very homogeneous path verifies that all the consecutive pixels along the path are resemblant. This suggests to measure the homogeneity by means of the aggregation, by means of a t-norm, of the resemblances between pairs of consecutive points in the path. We studied and compared different $t$-norms and in [18] we proposed to employ Weber's $t$-norms, whose expression is shown in equation 7 :

$$
W(a, b, \lambda)=\max \left\{0, \frac{a+b+a b \lambda-1}{1+\lambda}\right\}
$$

where $\lambda>-1, a$ is the result of the aggregation in the previous point and $b$ is the opposite value of the distance between the current point and the preceding one.

Depending on the value of parameter $\lambda$, this function offers homogeneity measures with different behaviors. This wide range of behaviors let us adapt the resulting membership function to the huge variety of regions that can be found in real images. In [18] we propose a technique to automatically find a value of $\lambda$ that can be used to calculate the fuzzy region in order to model a given region in the image, taking into account its characteristics.

\section{SignifiCANT REPRESENTATIVES OF FuZZy REgions}

With the methodology summarized in the previous section we can obtain a fuzzy region that contains full and exact information about the characteristics of a region. However, as it was mentioned in the introduction, in some applications a crisp result is needed. In this section we propose a methodology to obtain significant representatives of a fuzzy region as a subset of relevant $\alpha$-cuts.

\section{A. Crisp Representatives}

In fuzzy set theory it is usual to represent a fuzzy set by means of a collection of crisp sets, each one with an associated relevance degree. The standard representation for a fuzzy set is the set of its $\alpha$-cuts, where each $\alpha$-cut is a crisp approximation of the fuzzy set obtained by relaxing the membership restriction to a degree $\alpha$, allowing all the elements whose degree is greater or equal than $\alpha$ to be (fully) members of the set. Thus, we shall consider as possible crisp representatives of a fuzzy region $\widetilde{R}$ the different $\alpha$-cuts of $\widetilde{R}$, noted as ${ }^{\alpha} \widetilde{R}$, with $\alpha \in(0,1]$.

In practice, the number of different $\alpha$-cuts of a fuzzy region corresponds to the number of different membership degrees of pixels to the region. In most of the cases, this is a large number, yielding a large number of crisp representatives. But most of these possible representatives are quite similar, or correspond to a representation of the region that the user wouldn't perceive as significant or interesting. Such perception is related to the criterion employed in modeling the region, i.e., the homogeneity of the set of pixels that conform the crisp representative. On the basis of these intuitions, we propose the following definition of significant crisp representative:

Definition 3.1: Let $\Lambda(\widetilde{R})=\left\{\alpha_{1}, \ldots, \alpha_{n}\right\}$ be the set of positive membership degrees of $\widetilde{R}$, with $\alpha_{1}=1$ and $\alpha_{i}>$ $\alpha_{i+1} \forall i \in\{1, \ldots, n-1\}, \alpha_{n}>0$. An $\alpha$-cut $\alpha_{i} \widetilde{R}$, with $\alpha_{i} \in \Lambda(\widetilde{R})$, is a significant crisp representative of a fuzzy region $\widetilde{R}$ iff its homogeneity is significantly different from the homogeneity of $\alpha_{i-1} \widetilde{R}$.

The previous definition tries to capture the intuition that the borders of a crisp representative should perceptually correspond to those pixels where the homogeneity suffers a significant variation with respect to the previous ones, i.e., possible relative borders of the region to a certain degree. From now on, we will call relevant $\alpha$ values to those at which there is a significant change in the evolution of the homogeneity.

Now, our interest is to find a (small) set of $\alpha$-cuts corresponding to significant crisp representatives of a region. To comply with definition 3.1 , we need a way to measure the homogeneity of an $\alpha$-cut, and a way to determine when a change in homogeneity from one $\alpha$-cut to the next is significant. This is what we deal with in the next sections.

\section{B. Measuring the Homogeneity of an $\alpha$-cut}

Given a fuzzy region $\widetilde{R}$, we shall measure the homogeneity of an $\alpha$-cut $\alpha \widetilde{R}$ by means of a function $\mathcal{H}: \widetilde{\mathcal{P}}(\mathcal{I}) \times(0,1] \longrightarrow[0,1]$, where $\widetilde{\mathcal{P}}(\mathcal{I})$ is the set of all possible fuzzy subsets of pixels of an image $\mathcal{I}$, that should intuitively verify the following properties:

- If $\alpha=1$, then $\mathcal{H}(\widetilde{R}, \alpha)=1$.

- Monotonicity: if $\alpha_{i}>\alpha_{j}$ then $\mathcal{H}\left(\widetilde{R}, \alpha_{i}\right)>\mathcal{H}\left(\widetilde{R}, \alpha_{j}\right)$

With the first property we indicate that the kernel of the fuzzy set has maximum homogeneity; the second one means that as the value of $\alpha$ decreases, the homogeneity of the $\alpha$-cut should be lower.

The function $\mathcal{H}$ can be obtained as an aggregation of the membership degrees of pixels belonging to the $\alpha$-cut. The rationale behind this is that these degrees represent the homogeneity of the optimum path joining each pixel with the seed of the region, as explained in section II, equations 5 and 6. Therefore, this is a valid measure of the homogeneity of the region in every pixel. Following this idea, we propose to obtain the homogeneity measure as the average membership degree of the pixels belonging to the $\alpha$-cut, as follows: 


$$
\mathcal{H}(\widetilde{R}, \alpha)=\frac{\sum_{p \in^{\alpha} \widetilde{R}} \mu_{\widetilde{R}}(p)}{\left|{ }^{\alpha} \widetilde{R}\right|}
$$

where $\alpha \in(0,1]$. It is trivial to show that this measure verifies the properties we proposed at the beginning of this section.

\section{Finding the Significant Crisp Representatives}

By definition 3.1, an $\alpha$-cut is significant if there is a significant change of homogeneity with respect to the previous ones. The idea of change or variation can be expressed in terms of the gradient of the homogeneity measure evolution along the $\alpha$-cuts. In this paper, we calculate the gradient of the homogeneity measure along the set of $\alpha$-cuts by means of a discrete derivative of a gaussian function [7]. The local extremes of the gradient yield the place ( $\alpha$ values) and magnitude of the significant changes. This way, we can report the complete set of $\alpha$ values, ranked by the magnitude of the gradient, so that the user can take those that he/she consider more interesting.

If it is necessary to provide a small set of significant $\alpha$ values automatically, one possibility is to consider only variations with a magnitude over a relevance threshold $T_{\tilde{P}}^{\alpha}$. The final result depends obviously on the value $T_{\widetilde{R}}^{\alpha}$, to be fixed by the user. This lead us to the following definition:

Definition 3.2: Given a fuzzy region $\widetilde{R}$, we define the set of relevant $\alpha$ values, noted $\mathcal{A R}_{\widetilde{R}}$, as the set of $\alpha_{i} \in \Lambda \widetilde{R}$ corresponding to local extremes in the homogeneity gradient whose magnitude is greater than a given relevance threshold, $T_{\widetilde{R}}^{\alpha}$, plus $\alpha_{1}=1$ and $\alpha_{n}$.

In definition 3.2 we have included the values $\alpha_{1}=1$ and $\alpha_{n}$ because we consider the kernel and the support as significant representatives.

From definition 3.2 the set of relevant crisp representatives (relevant $\alpha$-cuts) is defined as follows:

Definition 3.3: Given a fuzzy region $\widetilde{R}$, we define the set of its significant $\alpha$-cuts, $\mathcal{A P R}_{\widetilde{R}}$, as the set of $\alpha$-cuts such that $\alpha$ is relevant, i.e.,

$$
\mathcal{A P}_{\widetilde{R}}=\left\{{ }^{\alpha} \widetilde{R} \mid \alpha \in \mathcal{A R}_{\widetilde{R}}\right\}
$$

In figure 2 (A) and (B) we can see, respectively, the original image, and the fuzzy region obtained applying the technique in section II to the seed marked with a cross in figure 2 (A). The evolution of the homogeneity measure for the $\alpha$-cuts can be seen as a blue line in figure 2 (C). Figure 2 (D) shows the gradient of the homogeneity, computed with a gaussian mask of size 3 and $\sigma=0.5$. In this two last figures the local extremes corresponding to relevant $\alpha$ values are marked with a pink square. The relevance threshold used, $T_{\tilde{R}}^{\alpha}$, is $40 \%$ of the highest local extreme value. Finally the $\alpha$-cuts representing the kernel and the support are marked in pink with triangle and a thick dotted line, respectively.

\section{RESULTS}

In this section we will show the results obtained by applying the technique proposed in previous section to the region
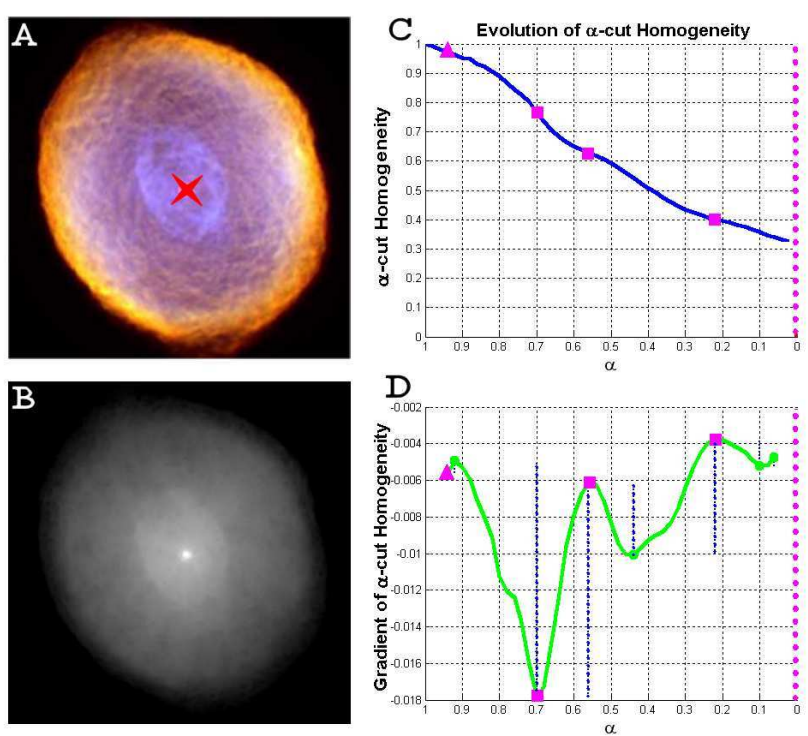

Fig. 2. Automatic selection of significant representatives of the fuzzy region. A: Original Image with a seed in the white center of the region. B: Fuzzy region obtained applying the technique in II. C: Evolution of the homogeneity along the sampled $\alpha$-cuts. $D: \alpha$-cuts homogeneity gradient and relevant $\alpha$ values.

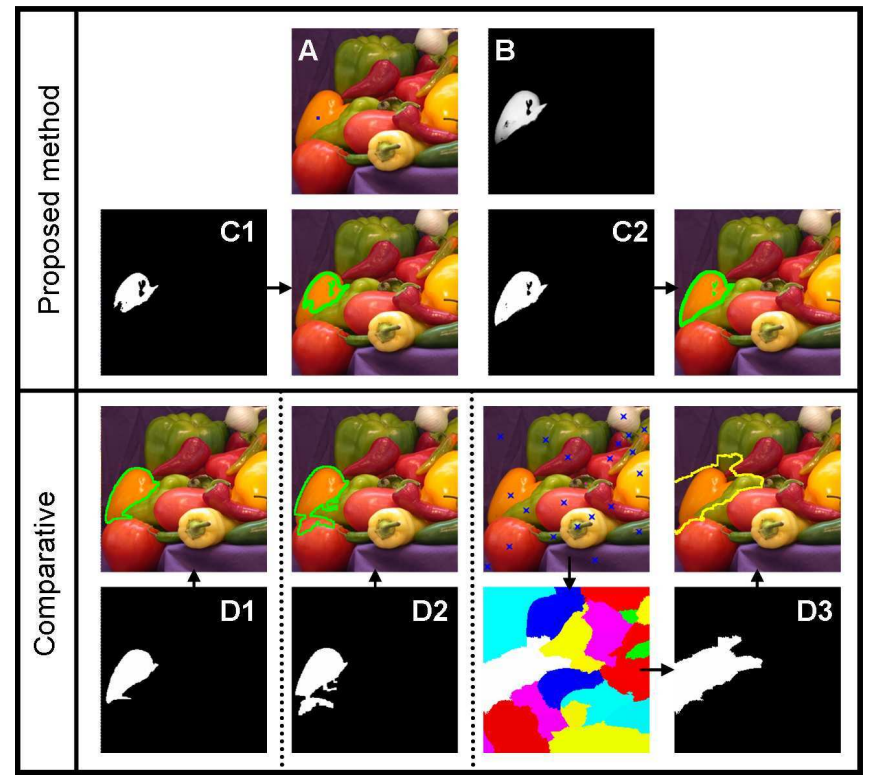

Fig. 3. A: Original Image with a seed marked with a dot. B: Fuzzy region obtained applying the technique in II. C1-2: Relevant crisp representatives corresponding to the relevant $\alpha$-cuts, and their crisp boundary superposed on the original image. D1: Crisp representative and boundary superposed resulting of Foliguet et al. [17] proposal. D2: Crisp region and boundary superposed resulting of Makrogiannis et al. crisp segmentation [13], [14]. D3: Crisp region resulting of a crisp region growing process with the marked set of seeds [7]. 


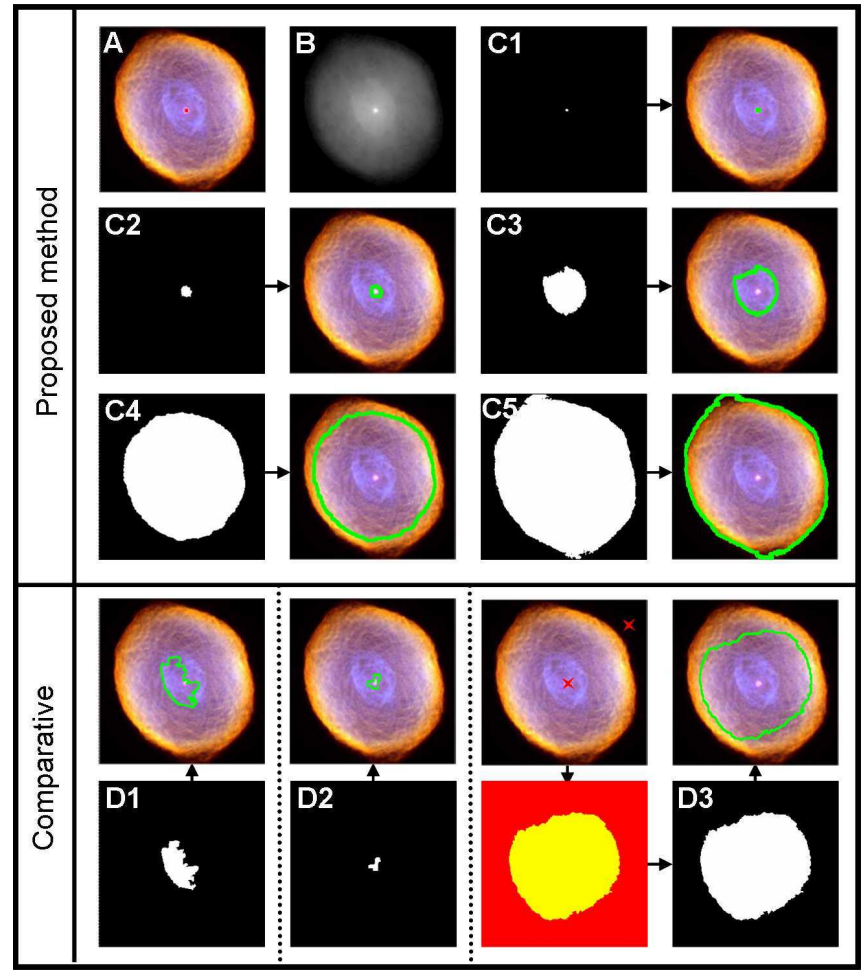

Fig. 4. A: Original Image with a seed marked with a dot. B: Fuzzy region obtained applying the technique in II. C1-5: Relevant crisp representatives corresponding to the relevant $\alpha$-cuts, and their crisp boundary superposed on the original image. D1: Crisp representative and boundary superposed resulting of Foliguet et al. [17] proposal. D2: Crisp region and boundary superposed resulting of Makrogiannis et al. crisp segmentation [13], [14]. D3: Crisp region resulting of a crisp region growing process with the marked set of seeds [7].

marked with a dot in figures 3(A), 4(A), and 5(A). In image 3(A) we have an almost crisp region with a soft shadow in the contour. In figure 4(A) there is a region with several concentric colors with soft and imprecise changes between them, and finally in figure 5(A) the region represents a light gradient.

Applying to these regions the technique in section II, we have obtained the fuzzy regions in figures 3(B), 4(B), and 5(B). Figures 3(C1-2), 4(C1-5) and $5(\mathrm{C} 1-4)$ show the relevant crisp representatives (relevant $\alpha$-cuts) found by applying our proposal on the corresponding fuzzy region, using the same sampling and threshold as in previous section. The contour of the crisp representative, superposed on the original image, is shown as well.

As figure 3 shows, when the region of interest is crisp, there are a few relevant $\alpha$-cuts that correspond to the most homogeneous area and to the whole region. However, in regions with many imprecision as in figures 4 and 5 there are more relevant crisp representatives. The first one corresponds to the most homogeneous area of the region, while the last one represents the support of the fuzzy region. Between them there are several $\alpha$-cuts corresponding to interesting crisp representatives, such us the different colors that represent the growth levels in the star, in figure 4, and the different

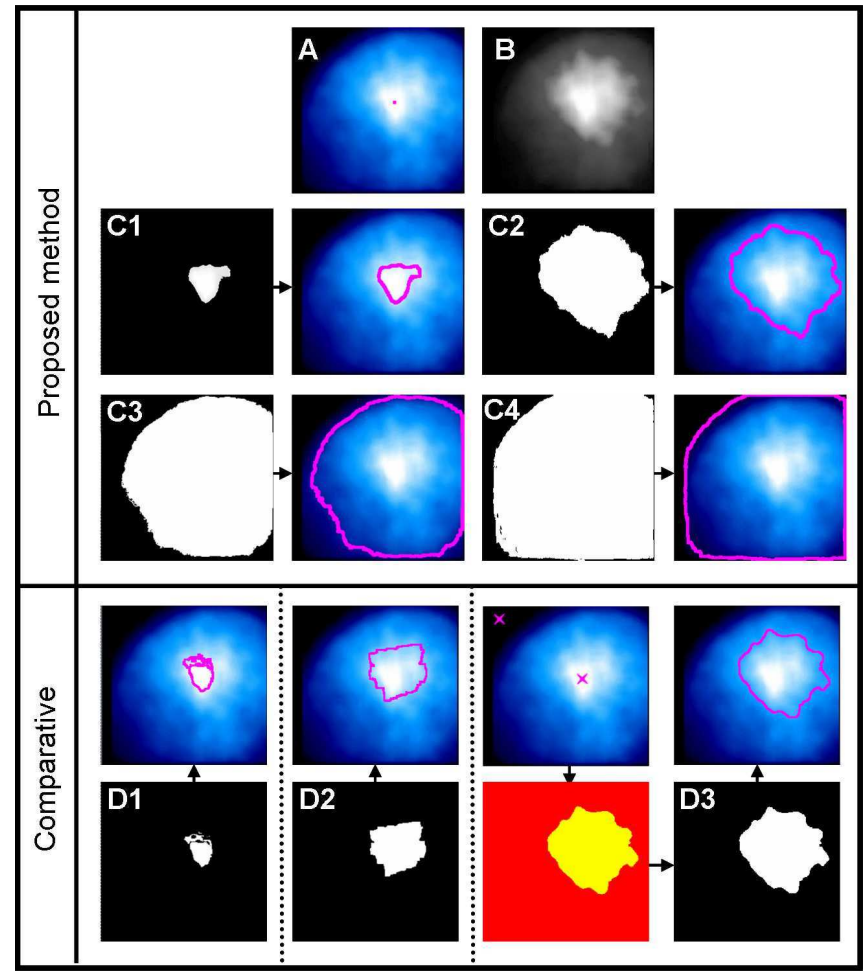

Fig. 5. A: Original Image with a seed marked with a dot. B: Fuzzy region obtained applying the technique in II. C1-4: Relevant crisp representatives corresponding to the relevant $\alpha$-cuts, and their crisp boundary superposed on the original image. D1: Crisp representative and boundary superposed resulting of Foliguet et al. [17] proposal. D2: Crisp region and boundary superposed resulting of Makrogiannis et al. crisp segmentation [13], [14]. D3: Crisp region resulting of a crisp region growing process with the marked set of seeds [7].

intensity levels in the comet, in figure 5. In the case of figure 4 , the relevant crisp representatives in images (C1) to (C5) correspond to the relevant $\alpha$ values marked in figure 2(C,D), from left to right.

In all the examples shown in this section, it can be appreciated that the relevant $\alpha$-cuts found correspond to changes in the homogeneity of the original region, and therefore to crisp representatives that most users would identify as relevant.

\section{A. Comparative}

Comparing crisp representatives of a fuzzy region with the results of other techniques is difficult. Mainly because with our proposal the result only depends on the fuzzy region obtained as starting point and the own technique, while the other proposals work with the whole segmentation of the image, and therefore the result is also conditioned by the adjacent regions (their seeds and growth).

Nevertheless we have searched for techniques with which the comparison may be representative: on the one hand we wanted to compare our results with those of another defuzzyfication technique; on the other hand, we also wanted to compare our crisp representatives with the result of region based crisp segmentation techniques. 
In figures 3, 4, and 5 we show the result of this comparison. The images (D) show the crisp result provided by other approaches, and the superposition of the boundaries on the original images. In the images (D1) of these figures we show the result of the technique proposed by Foliguet et al. [17]. They perform a fuzzy segmentation of the image based on a watershed algorithm. Once the fuzzy set for each region has been computed, they obtain a crisp approach by assigning each pixel in the image to the region to which it belongs with highest degree. In the images (D2) of these figures we have applied the segmentation algorithm proposed by Makrogiannis et al. [13], [14], that combines two region based techniques. Firstly they apply a watershed algorithm on the image, and then perform a fusion through the minimal expansion tree $(S S T)$, guiding the process with a region similarity measure. Finally in the images (D3) of these figures a classical region growing is employed [9].

As can be observed, the results obtained with the other techniques doesn't fit the region of interest, because of the aforementioned conditioning they suffer and the influence of neighbor regions. For example, in figure 3(D2-D3) the region for the orange pepper incorporates pixels corresponding to other regions, like the red pepper below in image 3(D2) and the green peppers and the background in image 3(D3), because the growth of a region doesn't stop until it reaches another region.

Looking at the comparatives in figures 4 and 5, where the boundaries of the regions are very imprecise, the methods employed in images (D1), (D2) and (D3) yield crisp regions that are clearly not representative of the region under study, in particular because they provide just a single representative. Several crisp approximations are needed in these images in order to obtain an acceptable solution. On the contrary, the proposed model allows us to achieve a good set of crisp representatives of the regions of interest.

\section{Conclusions}

In this paper we proposed a technique to obtain the set of most significant crisp representatives of a region in a color image. Starting from a seed pixel representative of the region of interest, a fuzzy model of the region is obtained by employing homogeneity measures suited to the characteristics of the region. The significant crisp representatives are obtained as those $\alpha$-cuts corresponding to significant variations of homogeneity.

Results obtained show that our technique works reasonably well in most of the cases. We have also shown the difficulties of trying to solve the same problem with approaches. Since the starting point is a seed, region growing techniques are to be employed. Both existing fuzzy and crisp techniques have the disadvantage that they need to fix at least two seeds in the image, i.e., they are designed to obtain a complete segmentation of the image. Hence, the result obtained (by applying another defuzzification method and other crisp region based segmentation techniques) depend on the position of other seeds. In addition, they provide a single crisp representative, hence losing many information. Though still losing information, obtaining a (small) set of crisp representatives is more informative. This is achieved by our technique, that also fits better the characteristics and contour of the region.

\section{REFERENCES}

[1] Amr R. Abdel-Dayem and Mahmoud R. El-Sakka Senior Member IEEE. Carotid artery ultrasound image segmentation using fuzzy region growing. Lecture Notes in Computer Science, 3656:869-878, October 2005.

[2] B.M. Cavalho, C.J. Gau, G.T. Herman, and T.Y. Kong. Algorithms for fuzzy segmentation. Proceedings of the International Conference on Advances in Pettern Recognition, pages 154-63, 1999.

[3] J. Chamorro-Martínez, D. Sánchez, B. Prados-Suarez, and E. GalánPerales. Fuzzy connectivity measures for path-based image segmentation. In IEEE International Conference on Fuzzy Systems, pages 218223. Reno, Nevada (USA), May 2005.

[4] J. Chamorro-Martínez, D. Sánchez, B. Prados-Suarez, and E. GalánPerales. Fuzzy homogeneity measures for path-based colour image segmentation. International Journal of Intelligent Systems Technologies and Applications, 1(3/4):301-318, 2006.

[5] Tie Qi Chen and Yi Lu. Color image segmentation - an innovative approach. Pattern Recognition, 35:395-405, 2002.

[6] M.C. Clark, L.O. Hall, D.B. Goldgof, L.P. Clarke, R.P. Velthuizen, and M.S. Silbiger. Mri segmentation using fuzzy clustering techniques. Engineering in Medicine and Biology Magazine, IEEE, 13(5):730-742, 1994.

[7] C.Russ. The Image Processing Handbook. CRC Press, 1994.

[8] A. Gillet, L. Macaire, C. Botte-Lococq, and J.G Postaire. Color image segmentation by fuzzy morphological transformation of the $3 \mathrm{~d}$ color histogram. Proceedings of the 10th IEEE International Conference on Fuzzy Systems, 2:824 -824, 2001.

[9] Gonzalez and Woods. Digital Image Processing. Prentice Hall, 2002.

[10] A.E. Hassanien, J.M. Ali, and H. Nobuhara. Detection of spiculated masses in mammograms based on fuzzy image processing. In Artificial Intelligence and Soft Computing ICAISC 2004, 2004.

[11] T.A. Kent and G.R. Hillman. A fuzzy rule based approach to identify brain structures using mri, in the presence of individual anatomical differences. In Fuzzy Information, 2004. Processing NAFIPS '04. IEEE Annual Meeting of the, volume 2, pages 1000-1001 Vol.2, 2004.

[12] J. Maeda, C. Ishikawa, S. Novianto, N. Tadehara, and Y. Suzuki. Rough and accurate segmentation of natural color images using fuzzy region-growing algorithm. In 15th International Conference on Pattern Recognition, volume 3, pages 638-641, October 2000.

[13] S. Makrogiannis, G. Economou, S. Fotopoulos, and N.-G. Bourbakis. Segmentation of color images using multiscale clustering and graph theoretic region synthesis. IEEE Transactions on Systems, Man \& Cybernetics, Part A Systems \& Humans, 35(2):224-38, 2005.

[14] Sokratis Makrogiannis, Iris Vanhamel, Spiros Fotopoulos, Hichem Sahli, and Jan P.H. Cornelis. Watershed-based multiscale segmentation method for color images using automated scale selection. Journal of Electronic Imaging, 14(3):033007 -, 2005.

[15] A. Moghaddamzadeh and N. Bourbakis. A fuzzy region growing approach for segmentation of color images. Pattern Recognition, 30(6):867-881, 1997.

[16] T.D. Pham. Image segmentation using probabilistic fuzzy c-means clustering. Proceedings of the International Conference on Image Processing, 1:722-725, 2001.

[17] S. Philipp-Foliguet, M.B. Vieira, and A. De Albuquerque Araujo. Segmentation into fuzzy regions using topographic distance. In Computer Graphics and Image Processing, 2001 Proceedings of XIV Brazilian Symposium on, pages 282-288, 2001.

[18] B. Prados-Suarez, J. Chamorro-Martínez, D. Sánchez, and J. Abad. Region-based fit of colour homogeneity measures for fuzzy image segmentation. Fuzzy Sets and Systems, 158(3):215-229, February 2007.

[19] Jar-Ferr Yang, Shu-Sheng Hao, and Pau-Choo Chung. Color image segmentation using fuzzy c-means and eigenspace projections. Signal Processing, 82:461-472, 2002.

[20] D.X. Zhong and H. Yan. Color image segmentation using color space analysis and fuzzy clustering. Proceedings of the 2000 IEEE Signal Processing Society Workshop, 2:624- 633, 2000. 